\title{
Severe hemolysis and vasoclusive crisis due to COVID-19 infection in a Sickle Cell Disease patient improved after Red Blood Cells exchange
}

\author{
Lina Okar ${ }^{1}$, Mohamad Rezek ${ }^{1}$, Amna Gamil ${ }^{1}$, Yahya Mulkandathil ${ }^{1}$, and mohamed yassin ${ }^{2}$ \\ ${ }^{1}$ Hamad Medical Corporation \\ ${ }^{2} \mathrm{HMC}$ NCCCR
}

January 7, 2021

\begin{abstract}
As the clinical course of COVID-19 infection in SCD patients is not clear, close monitoring is essential. We emphasize that RBC exchange should be offered early to avoid possible deterioration. We present a case of COVID-19 infection in a SCD patient causing severe hemolysis, that improved after RBC exchange.
\end{abstract}

\section{Introduction:}

Sickle Cell disease (SCD) is one of the most common hemoglobinopathies, it is due to mutation on the beta chain hemoglobin that causes deformity in red blood cells (RBCs) shape. The sickle shape of RBCs affects their function leading to hypoxia and sickle cell crisis including hemolysis when triggers like infections happen[1].

With the widely spread of Coronavirus infection, special population needs close monitoring as the exact clinical course and prognosis is not well known yet[2]. It was suggested that patients with SCD might present with vaso-occlusive crisis (VOCs) or acute chest syndrome (ACS) as first presentation of COVID19 infection[3]. The overlap between ACS and COVID-19 pneumonia made this an interesting area for research[4]. However, hemolytic crisis was not very common among previously reported cases[5].

Management plan for patients with SCD presenting with COVID-19 infection is not final yet[6]. However, following the recognized guideline for painful crisis, ACS and hemolysis treatment is essential. Previous studies showed the remarkable favorable impact of RBCs exchange in the course of COVID-19 infection among patients with hemoglobinopathies[7]. We believe it might prevent deterioration if offered earlier in the course of the disease. Treatment with hydroxyurea might or might not affect the susceptibility of COVID-19 infection[6]. Admission to the hospital is also required even if the cases are asymptomatic or mild because acute complications seems to happen after few days of infection.

Here we report a case of 48-year-old SCD patient compliant with HU who underwent post travel screening for COVID-19 infection and tested positive, admission and close monitoring were offered, he developed painful crisis and sever hemolysis after few days thus needed ICU admission and improved after RBCs exchange.

\section{Case presentation:}

A 48-year-old gentleman came back from a travel abroad and was tested positive for COVID-19 while screening him as part of post travel protocol. despite being asymptomatic, COVID-19 PCR from Nasopharyngeal swab was positive with a CT value 15 on 15 th November 2020. His past medical history was remarkable for Diabetes mellitus type II, on metformin and gliclazide, and Sickle Cell Disease on hydroxyurea $500 \mathrm{mg}$ BID. 
He had no previous surgeries. He has a history of recurrent pain crisis; most of them were managed in the Emergency department. His last blood transfusion in 2011. He has hypersplenism and avascular necrosis of the left shoulder, on conservative therapy. His last painful crisis was on 9the October 2020, for which he was managed with IV fluids, pain management, and discharged later in the same day. After testing positive for coronavirus, Labs (as shown in table 1) and Chest Xray (as shown in table 2) were ordered.

He was started on treatment; Favipiravir + Amoxicillin/Clavulanic acid for COVID-19 infection pneumonia, plus enoxaparin for deep vein thrombosis (DVT) prophylaxis. On day 4, he started to have intolerable back pain. Labs were repeated (Table 1) and the patient was transferred to ICU to manage his pain crisis. Repeated Chest X ray showed Interval progression of bilateral basal atelectasis and faint infiltrates more on the right side, which worsened later. Amoxicillin/Clavulanic acid was changed to Piperacillin/Tazobactam, hemoglobin was low (5.4) with hemolysis picture, Exchange transfusion was done for the patient, with 6 PRBCs. Dexamethasone was also added. The patient became stable after the transfusion and pain management. Another Chest $\mathrm{x}$ ray done 2 days later showed incomplete resolution of the widespread consolidation distributed over both lung fields when compared to a chest x-ray done 2 days ago his condition was improving. A repeated $\mathrm{HbS}$ was 22. 3 days later, he was discharged, with instructions about safety netting and home isolation, with resumption of his home medications. Throughout his stay in the Hospital, he did not need high oxygen flow nor intubation (the highest oxygen requirement was $1-2 \mathrm{~L}$ nasal cannula for one day).

\section{Discussion:}

Coronavirus family has multiple strain, the latest novel virus caused the coronavirus disease 2019 (COVID19) was called severe acute respiratory syndrome coronavirus 2 (SARS-CoV2)[8]. Data suggested that this new virus acts through specific receptors on the erythrocytes including ACE2, CD147 and CD26 causing pathological interaction with hemoglobin molecule and sometimes resulting in hemolysis[9].

Sickle cell disease (SCD) is a common inherited hemoglobinopathy. It is due to a single amino acid substitution in the sixth residue of beta globin subunit, this pathological change results in hemoglobin S (HBS). HBS is an abnormal hemoglobin that causes structure deformity in red blood cells (RBCs) changing it to crescent or sickle shape[10].

SCD complications are categorized into acute and chronic. The acute presentations are described as timedependent which mean there should be high suspension to diagnose them early and offer appropriate evaluation and treatment, thus improving the prognosis. These complications include ACS, vaso-oclusive crisis (VOC), acute anemia, hepatobiliary complications, Stroke, splenic sequestration, and priapism. Whereas chronic complications are pulmonary hypertension, hepatopathy due to iron overload, avascular necrosis, retinopathy, renal disease and peripheral ulcers [10].

Hemolytic crisis is defined as drop of more than or equal to $2 \mathrm{~g} / \mathrm{dL}$ from the baseline patient's hemoglobin value. Acute anemia might present as a single presentation or in accordance with splenomegaly or hepatomegaly [10]. However, when aplastic crisis diagnosis is made it is essential to rule out any possibility of delayed hemolytic transfusion reaction as more cautions is required if RBCs transfusion is the plan[1].

ACS is the most cause of hospital admission and mortality among SCD patients. The trigger for this condition is often infection, mostly bacterial and sometimes viral. Both ACS and COVID-19 pneumonia share a similar clinical feature such as fever, cough, shortness of breath and chest pain. Evidence from previous studies suggests that SARS-CoV2 could trigger ACS[11]. Due to this overlap between ACS and pneumonia, it is important to recognize the effect of COVID-19 in SCD patients[7].

Generally, patients with chronic comorbidities like diabetes, hypertension, lung diseases and cardiovascular diseases are at increased risk of COVID-19 complications. Noticeably, patients suffering from SCD have a lot of complications as mentioned before. In the presence of this multi-organ dysfunction,COVID-19 infection may trigger ACS or VOC[12]. Also, the abnormality in endothelial and procoagulants increase the risk of thromboembolic events, thus providing prophylactic anticoagulants is essential [2]. 
A recent retrospective study conducted in Bahrain reviewed 387 patients with SCD screened for COVID-19 infection, 6 patients had COVID-19 infection, all patients had favorable outcome, 3 patients were asymptomatic and 3 had mild to moderate symptoms. The study concluded that there is no difference among patients with SCD and non-SCD patients in term of COVID-19 clinical course, viral clearance, and infection rate. Also, no remarkable increased risk for SCD crisis[9]. In contrast to our case where the patient had asymptomatic presentation then developed acute painful episode with severe hemolysis. A case series of 10 patients with SCD infected with COVID-19 in UK also showed a good prognosis as 9 out of 10 patients recovered fully. Interestingly, 7 patients received blood transfusion (4 RBCs exchange and 3 simple transfusion). It is worthy to notice that one patients in this series died and she didn't receive blood transfusion as she had delayed hemolytic transfusion reaction as contraindication[13]. Another case series for 4 patients with SCD and COVID-19 infection showed that all patient presented with VOCs and had mild course, only one patient deteriorated and needed Intensive Care Unit (ICU) admission where the patient received RBCs exchange and improved remarkably. This case series suggest good prognosis of COVID-19 infection and emphasize on the importance of early RBCs exchange. However, none of the four patients mentioned in this case had acute hemolytic anemia[3]. Nino Balanchivadze et al published one center study that retrospectively reviewed 24 patients with SCD anemia or trait infected with COVID-19, 4 patients had SCD, none of the included patients was taking hydroxyurea (HU) the author concluded that patients with SCD generally had unremarkable course of disease, low possibility of ICU admission and intubation but hospitalization period might be longer [6]. It has been hypothised that COVID-19 infection in patients with SCD precipitate hemolysis because it affects hemoglobin beta chain, whereas others suggested that patients with Thalassemia and SCD have immunity against COVID-19 infection due to the defect in hemoglobin beta chain [9][14][15]. The physiology behind this hypothesis as published in one paper is that viral proteins like ORF1ab, ORF10 and ORF3a might cause porphyrin-associated iron molecule in heme to separate, this complex is located in the beta 1 chain, resulting in less amount of Oxygen being carried by the hemoglobin in the blood stream[15]. Patients with SCD as mentioned have mutation in beta chain hemoglobin, whether this plays a role in the protection against COVID-19 infection or not is still an area of research.

Red blood cells exchange is one way to treat severe ACS, a pervious data suggested its use in the case of severe COVID-19 pneumonia overlapping with ACS in patients with SCD. Moreover, it was noticed that whenever RBCs exchange offered earlier, the prognosis and clinic course were better[7][3]. In our case the patient improved after the red blood cells exchange and we suggest that it might play a role in the prevention of painful episode and hemolysis crisis progression if offered earlier in the course of infection.

While RBCs exchange form an important component in the acute management plan of SCD crisis, hydroxyurea is the mainstone in the maintenance therapy. However, it is not recognized yet if compliance with this therapy affects the presentation of COVID-19, data in this regard are conflicting, one retrospective study screened 40 patients with SCD for coronavirus infection, only 24 were positive and all of them were not on $\mathrm{HU}$, thus the authors suggested that $\mathrm{HU}$ in treatment might have a protective effect against the virus. This was not the case in our patients as he was on long term HU treatment and still had moderate course of illness[6].

Admission of Patients with SCD and COVID-19 infection is essential even if they are asymptomatic as they can develop acute painful episodes and hemolytic crisis later in the course of infection like our reported case. Although no final guideline is available now for the treatment of SCD patients with COVID-19 infection, we believe that offering RBCs exchange is an essential part of managing those population and we recommend to offer it as earlier as possible to avoid deterioration. Finally, the role of $\mathrm{HU}$ in the clinical course of coronavirus infection is still not defined and form an area for further research.

\section{Conclusion:}

Even though previous studies suggested favorable outcome for COVID-19 infection among patients with hemoglobinopathies. SCD remains at risk of acute deterioration even in the absence of any COVID-19 characteristic symptoms. Thus, close monitoring is necessary. ACS was mentioned before as a presentation of COVID-19 infection in those patients, yet acute hemolysis crisis should also be in mind. Offering RBCs 
exchange and admitting patients to the ICU until they are stable plays a role in improving prognosis and the earlier this is done the better the outcome. We believe that more studies are needed to form a clear guideline regarding RBCs exchange role and time of administration and the role of HU compliance on the course of infection.

\section{Acknowledgment}

We would like to acknowledge the Hematology section/ oncology department, Intensive care unit as well as Family medicine residency program at Hamad Medical Corporation for their support.

Open access fees offered by Qatar National Library (QNL).

\section{Statement of Ethics}

Consent was obtained from the patients.

Case approved by HMC Medical Research center

\section{Declaration of interest}

The authors report no conflicts of interest.

\section{Data availability statement:}

All data related to the article are available upon request.

\section{Authors contribution:}

Lina Okar and Mohamad Rezek wrote the manuscript.

Amna Gmeil, Yahya Mulikandayhil, and Mohamed A. Yassin reviewed the literature and reviewed the manuscript.

\section{References:}

[1] Wali Y, Kini V, Yassin MA. Distribution of sickle cell disease and assessment of risk factors based on transcranial Doppler values in the Gulf region. Hematology. 2020 Jan 1;25(1):55-62.

[2] Menapace LA, Thein SL. COVID-19 and sickle cell disease. Haematologica 2020;105:2501-4. https://doi.org/10.3324/haematol.2020.255398.

[3] de Sanctis V, Canatan D, Corrons JL, Karimi M, Daar S, Kattamis C, Soliman AT, Wali Y, Alkindi S, Huseynov V, Nasibova A. Preliminary Data on COVID-19 in patients with hemoglobinopathies: A multicentre ICET-A study. Mediterranean Journal of Hematology and Infectious Diseases. 2020;12(1).

[4] Beerkens F, John M, Puliafito B, Corbett V, Edwards C, Tremblay D. COVID-19 pneumonia as a cause of acute chest syndrome in an adult sickle cell patient. Am J Hematol 2020;95:E154-6. https://doi.org/10.1002/ajh.25809.

[5] Chakravorty S, Padmore-Payne G, Ike F, Tshibangu V, Graham C, Rees D, et al. COVID-19 in patients with sickle cell disease - A case series from a UK tertiary hospital. Haematologica 2020;105:2691-3. https://doi.org/10.3324/haematol.2020.254250.

[6] Balanchivadze N, Kudirka AA, Askar S, Almadhoun K, Kuriakose P, Fadel R, et al. Impact of COVID19 Infection on 24 Patients with Sickle Cell Disease. One Center Urban Experience, Detroit, MI, USA. Hemoglobin 2020. https://doi.org/10.1080/03630269.2020.1797775.

[7] Okar L, Aldeeb M, Yassin MA. The role of red blood cell exchange in sickle cell disease in patient with COVID-19 infection and pulmonary infiltrates. Clin Case Reports 2020:ccr3.3526. https://doi.org/10.1002/ccr3.3526. 
[8] Gorbalenya AE, Baker SC, Baric RS, de Groot RJ, Drosten C, Gulyaeva AA, et al. The species Severe acute respiratory syndrome-related coronavirus: classifying 2019-nCoV and naming it SARS-CoV-2. Nat Microbiol 2020;5:536-44. https://doi.org/10.1038/s41564-020-0695-z.

[9] AbdulRahman A, AlAli S, Yaghi O, Shabaan M, Otoom S, Atkin SL, et al. COVID-19 and sickle cell disease in Bahrain. Int J Infect Dis 2020;101:14-6. https://doi.org/10.1016/j.ijid.2020.09.1433.

[10] Yassin MA, Soliman AT, de Sanctis V, Abdula MA, Riaz LM, Ghori FF, Yousaf A, Nashwan AJ, Abusamaan S, Moustafa A, Kohla S. Statural growth and prevalence of endocrinopathies in relation to liver iron content (LIC) in adult patients with beta thalassemia major (BTM) and sickle cell disease (SCD). Acta Bio Medica: Atenei Parmensis. 2018;89(Suppl 2):33.

[11] Sivalingam T, Inusa B, Doyle P, Oteng-Ntim E. COVID-19 and the pulmonary complications of sickle cell disease. EJHaem 2020:545-7. https://doi.org/10.1002/jha2.105.

[12] Sheha D, El-Shayeb M, Eid Y, Amin M, Saeed A, Abdou D, et al. Unfolding of sickle cell trait by coronavirus disease 2019 (COVID-19) infection. $\mathrm{Br}$ J Haematol 2020;191:e38-40. https://doi.org/10.1111/bjh.17089.

[13] London S, London S. Ase eports 2020;105:2691-3.

[14] Torti L, Maffei L, Sorrentino F, De Fabritiis P, Miceli R, Abruzzese E. Impact of SARS CoV-2 in hemoglobinopathies: a protective mechanism being from Beta chain Hemoglobin defects? Mediterr J Hematol Infect Dis 2020;12:e2020052. https://doi.org/10.4084/mjhid.2020.052.

[15] wenzhong liu, hualan L. COVID-19: Attacks the 1-Beta Chain of Hemoglobin and Captures the Porphyrin to Inhibit Human Heme Metabolism 2020. https://doi.org/10.26434/CHEMRXIV.11938173.V7.

\section{List of figure and table legends:}

Table.1 Laboratory results upon admission, deterioration and discharge.

Table 2. Chest Xrays. 